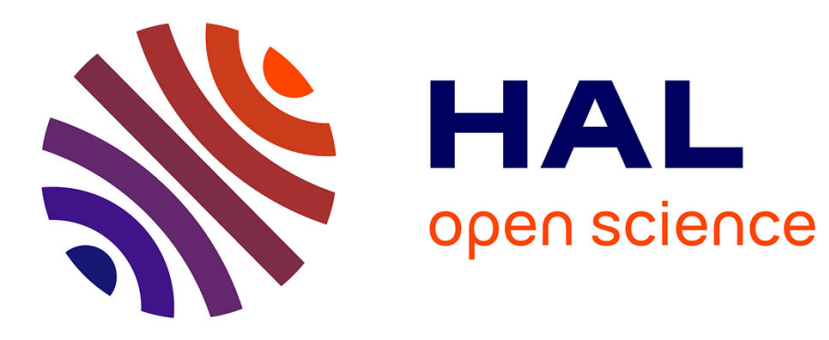

\title{
Geomagnetic effects observed by the Codalema experiment
}

Lilian Martin, Collaboration Codalema

\section{To cite this version:}

Lilian Martin, Collaboration Codalema. Geomagnetic effects observed by the Codalema experiment. Nuclear Instruments and Methods in Physics Research Section A: Accelerators, Spectrometers, Detectors and Associated Equipment, 2011, 630 (1), pp.177-182. 10.1016/j.nima.2010.06.059 . hal00438151

\section{HAL Id: hal-00438151 \\ https://hal.science/hal-00438151}

Submitted on 2 Dec 2009

HAL is a multi-disciplinary open access archive for the deposit and dissemination of scientific research documents, whether they are published or not. The documents may come from teaching and research institutions in France or abroad, or from public or private research centers.
L'archive ouverte pluridisciplinaire HAL, est destinée au dépôt et à la diffusion de documents scientifiques de niveau recherche, publiés ou non, émanant des établissements d'enseignement et de recherche français ou étrangers, des laboratoires publics ou privés. 


\title{
Geomagnetic effects observed by the Codalema experiment
}

\author{
L.Martin \\ SUBATECH, Université de Nantes/Ecole des Mines de Nantes/IN2P3-CNRS, Nantes, France \\ for the CODALEMA collaboration \\ GSII, ESEO, Angers, France \\ $L A L$, Université de Paris Sud/IN2P3-CNRS, Orsay, France \\ $L A O B$, Université de Besançon/INSU-CNRS, Besançon, France \\ LESIA, USN de Nançay, Observatoire de Paris-Meudon/INSU-CNRS, Meudon, France \\ $L P C E$, Université d'Orléans/INSU-CNRS, Orléans, France \\ LPSC, Université Joseph Fourier/INPG/IN2P3-CNRS, Grenoble, France
}

\begin{abstract}
The CODALEMA experiment is measuring transient radio emissions associated to extended air showers produced by high energy cosmic rays. The experimental setup installed at the Nançay Radio Observatory in France has recently undergone hardware upgrades and an extension of the surfaces covered by both the antenna and the scintillator detector arrays. The experimental data allow to investigate the main features of these radio signals and the underlying electric field production mechanisms. Some of the latest experimental results of CODALEMA are presented. They have been analyzed assuming a linear dependence of the electric field with respect to $v \wedge B$. Within the CODALEMA observation conditions at Nançay, the detection efficiency, the arrival direction distribution and the polarity of the radio signals can be interpreted in terms of a geomagnetic effect. A R\&D effort is currently underway to develop the hardware elements for the deployment of a large detector array based on active antennas. The main features of the first prototype of the CODALEMA autonomous station are briefly described.
\end{abstract}

Key words: Extensive air showers, Ultra high energy cosmic rays, Radio detection, CODALEMA

\section{Introduction}

Height French laboratories are forming the CODALEMA collaboration interested in measuring the radio signal associated to the extended atmospheric shower (EAS) produced by highly energetic cosmic rays reaching the Earth. By revisiting a technique investigated almost 50 years ago, the collaboration aims at understanding the radio signal production mechanisms and at identifying key observables correlated to the air shower and the primary particle features. From an experimental point of view, the collaboration is pursuing the objective of developing a detection technique competitive with conventional surface detectors (Cerenkov and scintillator detector or fluorescence telescope) in terms of quality of data (sensitivity and resolution), efficiency and duty cycle, simplicity, robustness and cost. This latest criterion becomes a major element in the perspective of the construction of a ground detection array over an extremely large area.

\section{Experimental setup}

The CODALEMA experimental setup has significantly evolved since the first attempts started in 2002 to measure radio signals with logarithmic antennas from the Nançay

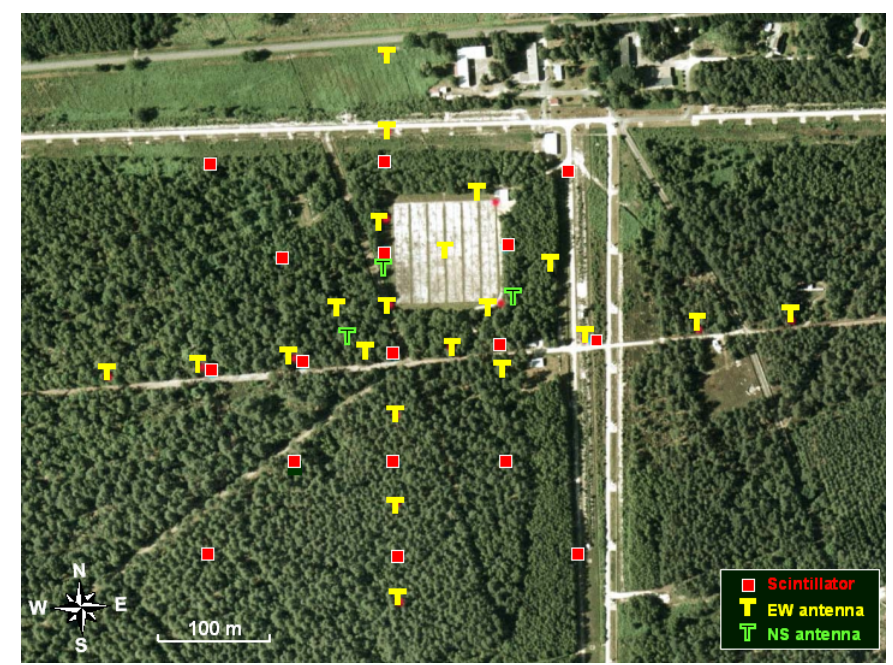

Figure 1: Aerial view of the CODALEMA experimental site.The locations of scintillator detectors (squares) and antennas $(\mathrm{T})$ are shown. The DAM is the gray area at the center and is covering a $80 \times 80 m^{2}$ area. 


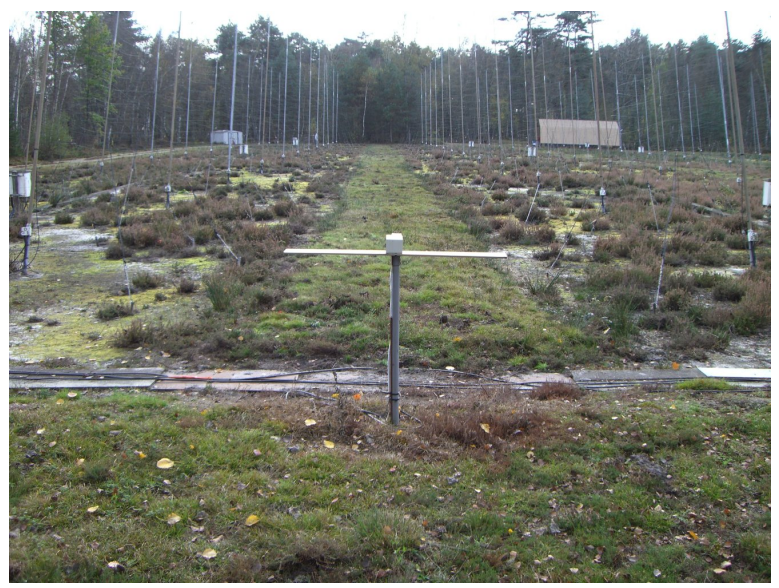

Figure 2: One of the short dipole antenna in front of the logarithmic antennas of the DAM. The shelter housing the detector power supplies and the DAQ system appears at the back left of the picture.

Decametric Array (DAM) [1]. The small group of logperiodic antennas has been progressively extended [2] and then replaced by fat active dipoles. The original filtered antenna self triggering the whole antenna array has been superseded by a rudimentary group of four scintillator detectors itself upgraded and extended in 2006 by a new surface detector array [3]. The current setup CODALEMA experimental setup is made of three overlapping apparatus : an array of 17 surface detectors, the 144 logarithmic antennas of the DAM and and larger array of short dipole antennas (figure 1).

The ground particle array is dedicated to the characterization of the arrival time, direction, size and core location of the shower by a measurement of the particle density at the ground level [3]. Moreover it provides a logic signal to trigger the antenna digitization and acquisition systems. It consists in 17 scintillator stations located on a grid of an approximate spacing of $85 \mathrm{~m}$. It covers a $340 \times 340 \mathrm{~m}^{2}$ surface whose center roughly matches the radio array center. Each station includes a thick plastic scintillator seen by two photomultipliers, all inserted in stainless steel box, finally housed in a plastic container for weather protection. The two photomultipliers have their high voltage supply set to work at different gains (high gain (HG) and low gain $(\mathrm{LG}))$, in such a way to have an overall dynamics from 0.3 to 3000 vertical equivalent muon (VEM).

The core of the radio array is made of 14 antennas, around $90 \mathrm{~m}$ spaced, and forming a cross of two $600 \mathrm{~m}$ long arms oriented in the North-South and East-West directions. These antennas are themselves oriented in the East-West direction. 7 antennas were recently added in order to sample the radio signal associated to EAS in a diagonal direction and at smaller distance. 3 dipoles were installed near the array center, close to existing antennas and oriented in the North-South direction to study the polarization of the electric field.

Despites unquestionable advantages, the large size of the logarithmic antenna prevents their deployment over a large area. Thus a short active dipole design has been followed to meet criteria of size, performance, simplicity and cost. It is made of two $0.6 \mathrm{~m}$ long and $0.1 \mathrm{~m}$ wide aluminum slats, separated by a $10 \mathrm{~mm}$ gap. It is hold horizontally at $1 \mathrm{~m}$ above ground by a plastic mast (figure 2 ). The antenna radiator length results in a resonating behavior around 115 $\mathrm{MHz}$. This antenna is loaded by a high input impedance dedicated low noise $34 \mathrm{~dB}$ amplifier whose $3 d B$ bandwidth is $100 \mathrm{kHz}-220 \mathrm{MHz}$. In the working frequency band the antenna directivity stays almost isotropic and varies rather smoothly with the frequency[4].

All the detectors and antennas are wired to a central shelter that protects power supplies, racks of electronics and computers for data taking. In the standard acquisition mode, the particle detection system acts as a master EAS trigger while the antennas are configured in a slave mode.

Signals from both array detectors are directed to 4 channel $6 \mathrm{U}$ VME waveform digitizer boards. The so-called Matacq board performs a fast 12-bits digitalization of the waveforms with $300 \mathrm{MHz}$ analog bandwidth at a sampling rate set to $1 \mathrm{Gs} / \mathrm{s}$ and in a memory depth of 2560 points (2.5ms of signal). The maximum excursion at $1 \mathrm{~V}$ analog input of these ADCs defines a LSB at $250 \mathrm{mV}$. The noise of the antenna chain (antenna + preamplifier + cable) measured at the input of the digitizer is less than $200 m V_{r m s}$.

All the ADC boards are externally triggered by a dedicated 16-fold multiplicity circuit. This circuit discriminates the HG photomultiplier signals with a threshold corresponding to $0.3 \mathrm{VEM}$ and compares the resulting multiplicity to a remotely controlled level. In standard data taking conditions, we requires the 5 central stations to trigger within a $600 n s$ gate width. These trigger conditions lead to an event rate of about 200 events/day.

The data acquisition software is running under the LabView environment installed on two conventional commercial PC. Subroutines have been developed to interact with the Matacq boards via GPIB interfaces allowing to initialize and program the boards, calibrate and readout the data. On line monitoring and data transmission over the network for storage and further processing are also performed from those computers.

\section{Offline data processing}

The offline processings of the data measured by the surface detectors and the antennas are rather similar [5]. Tagging procedures are used to determine time and amplitude in each individual device. Arrival directions are then determined in both arrays by computing the time difference of arrival of the received signals. By comparing the signal amplitudes in the detectors and assuming a given form of the lateral distribution, the shower core position can be estimated and the variation of the signal with respect the the shower axis can be studied.

In addition, the SD amplitudes can be further processed to determine a vertical shower size (using a Constant Intensity Cut method) itself correlated to the energy 

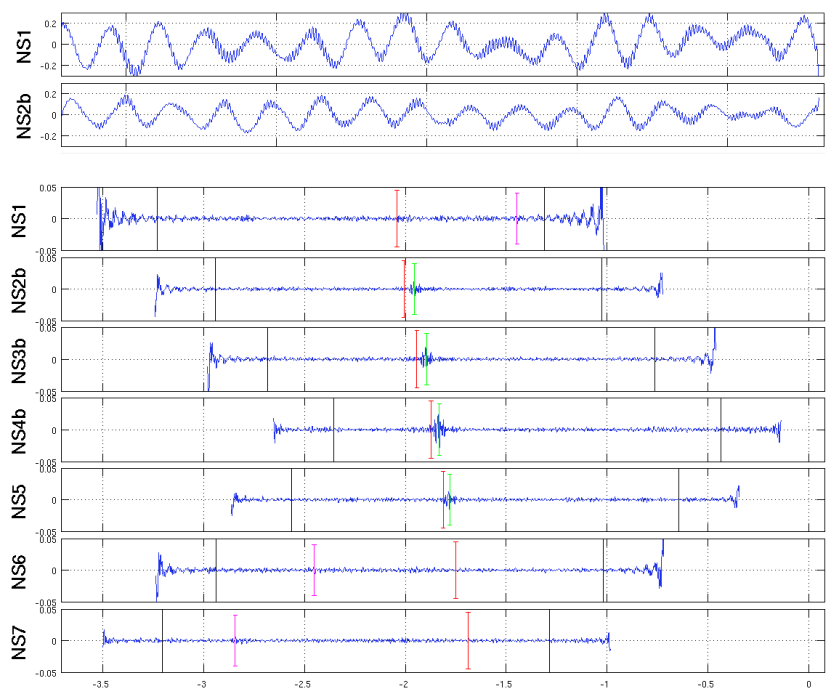

Figure 3: Offline processing of the antenna time signals (in $\mu s$ ). Raw signal of two antennas (top) and filtered signals $(23-82 \mathrm{MHz}+$ $110-130 \mathrm{M} \mathrm{Hz}$ ) in the antennas of the NS arm of the array (bottom) are shown. An vertical line superimposed on the signal indicates a tagged antenna. The predictions from the SD reconstruction are also shown as vertical lines (slightly shifted in time)

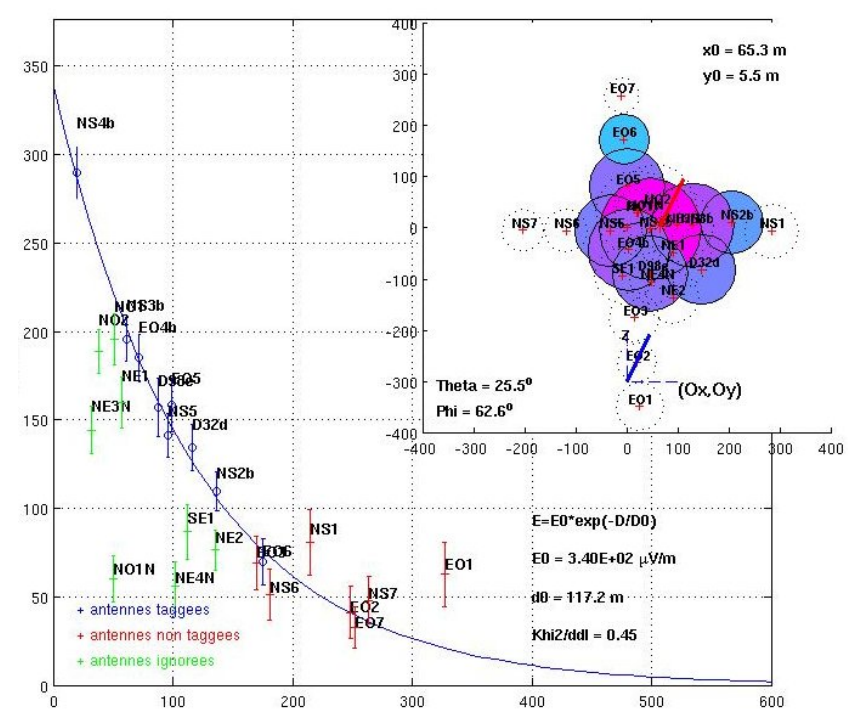

Figure 4: Radio amplitude (in u.a.) distribution as a function of the distance to the shower axis (in $\mathrm{m}$ ). Tagged antenna (circle) are used in the exponential fit. Antennas ignored or without a tagged signal are also indicated by simple vertical error bars. Footprint on the antenna array is shown (top right) where the circle surfaces are proportional to the signal amplitudes.

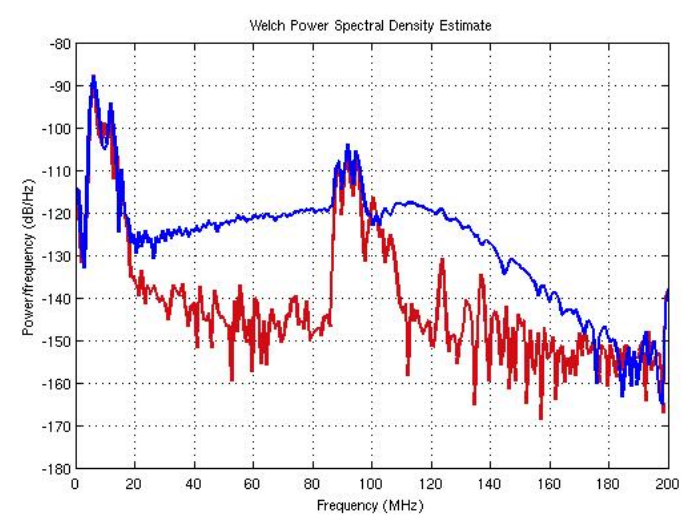

Figure 5: Frequency spectrum observed in an antenna for a very large event (top curve) compared to the spectrum observed for usual events (bottom curve). One should note that the EAS radio counterpart extends at frequencies well higher the FM and presents a power spectral density competitive to the FM band.

of a primary proton with the help of AIRES simulations. At $10^{17} \mathrm{eV}$ the uncertainty on the energy has been estimated around $30 \%$ assuming protons as primary particles.

On the contrary to the signals measured in scintillator detectors, the antenna signals need a filtering processing before the tagging step. As shown on the figure 3, contributions from the AM and FM emitters are dominating in the raw signals which prevent direct observations of the radio transient signal in the antenna data. Frequency filtering (typically in between these two anthropic radio bands) allows to identify clearly radio counterparts of the EAS. A specific procedure has been recently developed for the tagging of the antenna signals. Based on a linear prediction method, it improves the cosmic radio pulse recognition efficiency in the presence of sporadic emitters.

Typical events exhibit a radio signal in a restricted number of antennas (fig.3). Clear variations can be observed in their lateral distributions with respect to the shower axis (fig.4). Their frequency spectra however are dominated by the AM and FM bands and do not show any particular contribution (fig.5). These events are usually characterized by a low energy as estimated by the scintillator detectors. In several case however the event displays a time signal discernible in the raw signal and a spectrum featuring a very broad band signal (fig.5). Most of the time, in that case the maximum number of tags is reached in the antenna array.

The last step of the offline data processing is dedicated to the event selection. Based on the arrival times and directions estimated in both arrays, coincidence criteria are applied to select well reconstructed events. The arrival directions should have an angular difference smaller than $20^{\circ}$ and the arrival time difference should be smaller that 100ns. For the following analysis, the events are sorted in two classes depending on the scintillator informations. Internal events include events whose station with the maximum signal is not on one edge of the SD array. For these 


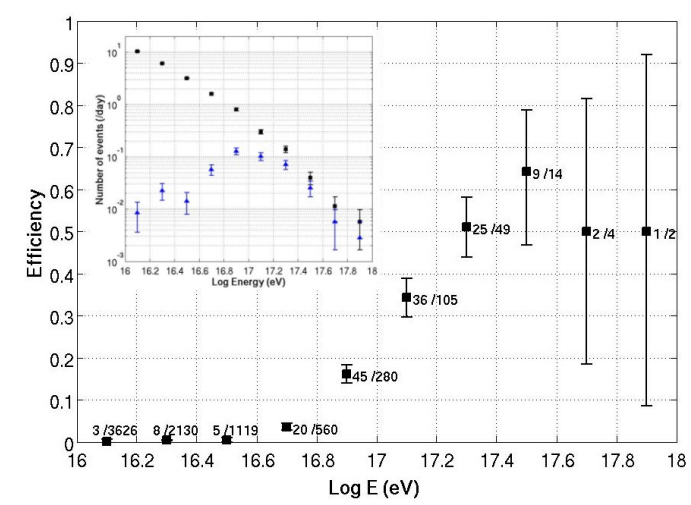

Figure 6: The radio detection efficiency as function of the energy. This curve is calculated from the number of count distributions (insert) of the internal events recorded in the SD (circles) and in coincidence in the antennas (triangles).

events a correct estimation of the shower energy and core position is expected. For the other events (external events) the arrival direction is correct but the energy and axis position values are unreliable.

\section{Physics results}

A set of data taken between November 2006 and March 2008 has been processed, cumulated and summarized in the table 1 . The large difference between the number of counts in the SD and in the antenna arrays illustrates the very different energy threshold of these apparatus in their current configurations. About one coincident internal event every two days are recorded while the rate of coincidence events is 4 times higher. This difference has been reduced later by extending the SD array from 13 to 17 stations. The 4 new stations have been installed at the corners of the array allowing this way to enrich the fraction of internal event in the data sample.

\begin{tabular}{|c|c|}
\hline Effective time & 355 days \\
\hline Triggers (SD events) & 61500 \\
Reconstructed antenna events & 750 \\
Coincidences (SD and antenna) & 620 \\
Coincidences (internal) & 157 \\
\hline
\end{tabular}

Table 1: Statistics of the data set recorded between March 2006 and November 2008 and used for the analysis presented in this paper.

The energy spectrum of the coincident internal events is compared to the spectrum of internal events recorded in the scintillator detectors (no coincidence criteria applied) in the figure 6 . The radio detection efficiency is calculated from these distributions. One can deduce a radio detection threshold around of $5 \cdot 10^{16} \mathrm{eV}$ of the CODALEMA experiment. The efficiency curve regularly increases with increasing energy but does not clearly reaches a full efficiency in the observed energy range.

This feature of the efficiency curve can be understood by looking at the angular distribution of the arrival di- rection of the coincident events observed (figure 7). This distribution clearly exhibits a large asymmetry in the azimuthal direction. Most of the events observed in the antenna array is coming from North while the Southern hemisphere is sporadically and irregularly populated. The ratio of the number of events coming from the South $\left(90^{\circ}<\phi<\right.$ $\left.270^{\circ}\right)$ to the total number of events has been evaluated to $0.17 \pm 0.02$. Detailed analysis have been performed to rule out a statical effect or an antenna array acceptance limitation to explain this azimuthal anisotropy. Moreover it has been shown that this observed deviation from symmetry tends to disappear with increasing energy signing a threshold effect [5].

The observed asymmetry has been analyzed with the hypothesis that the geomagnetic field is responsible for the symmetry breaking in the electric field generation [5]. As a matter of fact, we assumed that the Lorentz force acting on moving charged particles induced by the geomagnetic field is the driving element of the emission mechanism producing the electric field measured by the antennas. In our analysis, the amplitude of electric field is thus considered as proportional to the vector cross product $v \wedge B$ where $v$ is the direction of the shower axis and $B$ the Earth magnetic field at the location of the experiment. Moreover we assume that the electric field polarization is linear and oriented along $v \wedge B$. Finally as we are working at the radio detection threshold, we are making the last hypothesis that the number of radio events is simply proportional the signal amplitude. A predicted event sky map can thus be computed by calculating the amplitude of the Lorentz force component projected on the East-West axis convoluted with both the scintillator detector acceptance and the antenna reception diagrams.

This prediction appears to be very similar to the observed sky map especially since it reproduces the main feature of the experimental distribution. The agreement can be quantitatively assessed by looking at the zenithal and azimuthal angular distributions (figure 8). The locations and relative magnitudes of the maxima and minima in the azimuthal distribution are rather well reproduced.

A similar analysis has been performed on data recorded by the three antenna recently added and oriented in the North-South direction. With a restricted data sample, preliminary results confirm the interpretation of the angular distribution of arrival directions in terms of $v \wedge B$. The observed sky map is shown in figure 9 and features a rather symmetric distribution with respect to the NS and the EO axis. A lack of event is now observed in the North and South directions. A prediction sky map of the $v \wedge B$ dependence projected on the NS polarization has been computed and compared with the experimental azimuthal distribution (figure 9).

The interpretation of in term of $v \wedge B$ cross product has been pushed further by comparing not only the amplitude of the electric field to the observed number of counts but also by comparing the polarity of the vector cross product to the sign of the radio signals (averaged over the tagged 


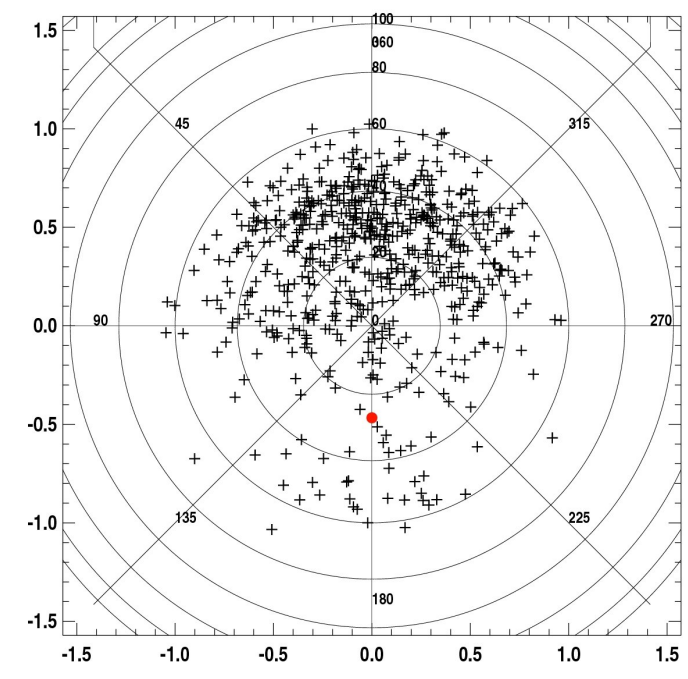

Figure 7: Sky map of radio events observed by the EW antenna array. The zenith is at the center, the azimuth is: North (top, $0^{\circ}$ ), West (left, $90^{\circ}$ ), South (bottom, $180^{\circ}$ ) and East (right, $270^{\circ}$ ); the direction of the geomagnetic field at Nançay is indicated by the dot.

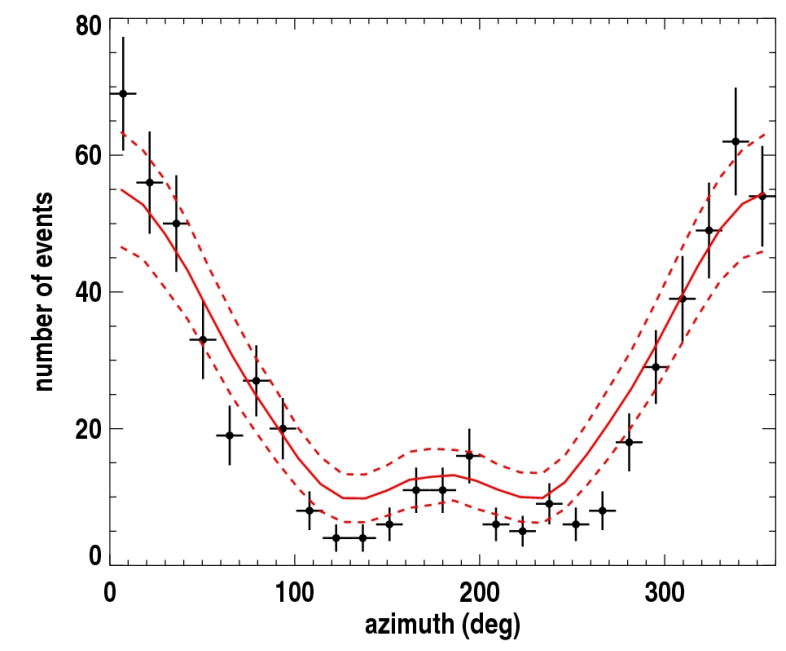

Figure 8: Azimuthal distribution of events observed by the EW antenna array. The solid line represents the predicted distribution obtained from simulated events according to the predicted coverage map. The dashed lines define the $\pm 1 \sigma$ band around the prediction.
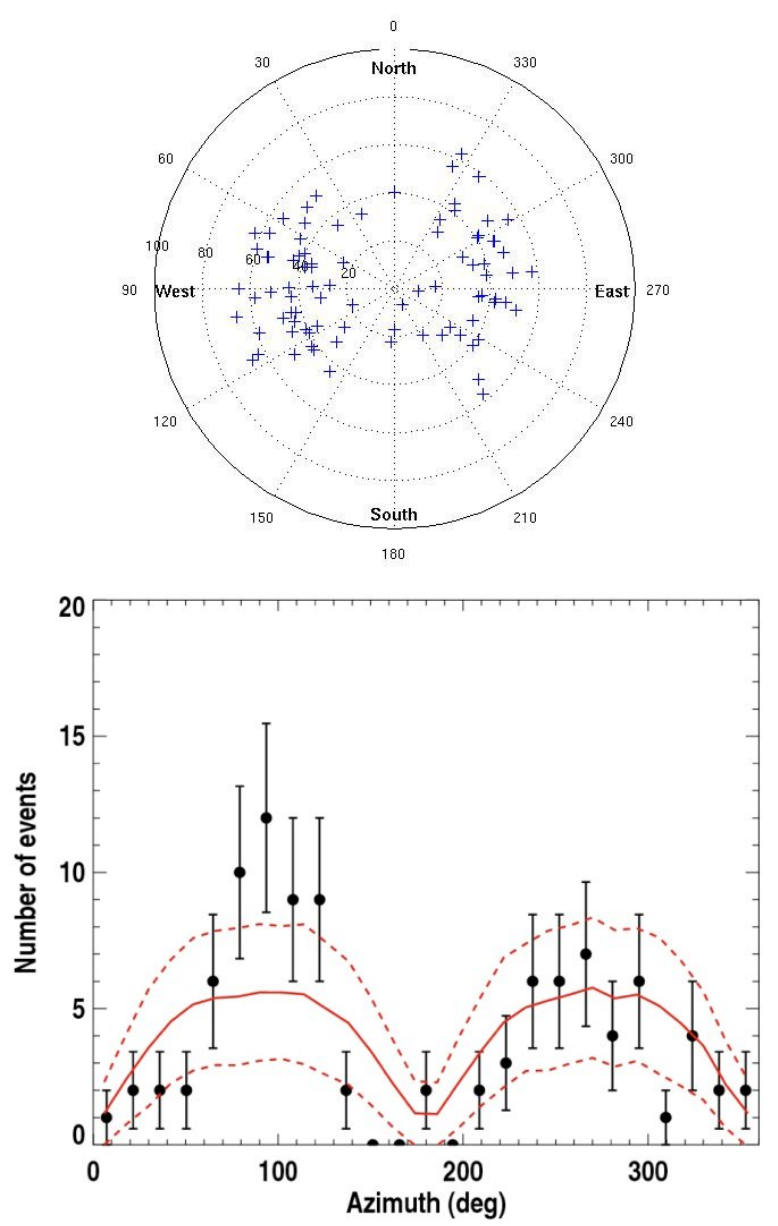

Figure 9: Sky map of the events observed by the small NS oriented antennas (top). Azimuthal distribution of events measured by the NS antenna mini-array (bottom). Definitions of the lines are similar to those in the figure 8 .

antennas). As predicted by the $v \wedge B$ scenario, the sign of the events are in opposition in the North and the South hemispheres for events measured with the EW antenna[5]. An identical study has been done for the set of data measured by the mini-array of NS antennas which shows a sign inversion between events observed from the West and East directions which again matches the prediction of a polarity flip.

These analysis are currently being pursued and completed by the new set of data taken during almost a full year. The lateral distributions of the radio electric field are now the subject of a detailed study in order to extract a precise correlation between the amplitude of the observed electric field and the estimated energy of the primary particle given by the surface detectors.

\section{Toward a new station}

The CODALEMA experiment is running and taking data without any major interruption or extended failures since October 2006. These stable running conditions were 


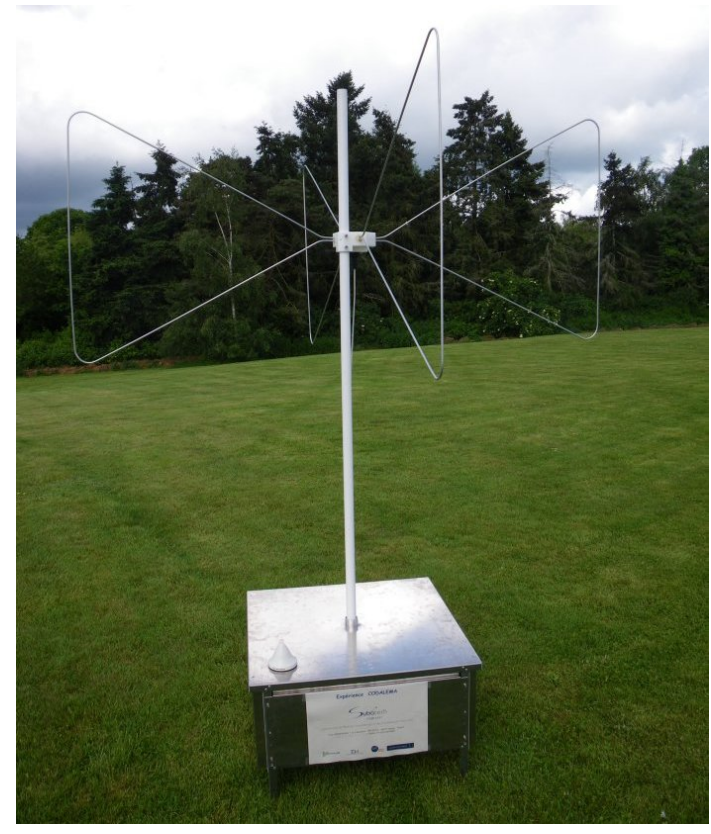

Figure 10: The new autonomous station. It features a dual polarization antennas hold by a container hosting the on board electronics.

required in order to cumulate large statistics of events and to allow refined analysis of radio signals associated to EAS. Extending further out the CODALEMA setup at Nançay requires a complete change in the installation strategy. The current strategy (all detection devices connected by underground cables to the central container) clearly reaches its limits in terms of infrastructure workload and cost and in terms of time propagation and attenuation of the signal in the cables. New radio station prototypes are currently built and feature a trigger system, power supply and data transfer autonomous from the wired arrays and able to work in a standalone mode. Some criteria were considered when designing the station structure and its components : the use of commercial technologies and standard protocol, the use of modular functions, a station quiet in terms of EMI with a low overall power consumption, a remote control of settings and an competitive overall cost competitive.

Regular Ethernet connections are used to link the antenna to the electronic boards and further out for communication between electronic modules. Radio signals from the antennas are sent to a trigger board where they are narrow band filtered and compared to remotely adjustable analog threshold. Large band signals are read by a digitizer board (a new version of the Matacq board) featuring a circular analog memory and a flash ADC with a 14 bit dynamic. The time tagging of events is made by a GPS board equipped with a Motorola M12T device. The local acquisition is running on an on board PC which serves as local storage for the data, manages the communications from and to the outer word and can be used to run higher sophisticated trigger level. The PC and the other boards are interfaced by a Rabbit controller using a TCP/IP pro- tocol. All these elements fit in a crate itself installed in a metallic box designed for weather protection and electric shielding. A specific attention has been payed to the compartment hosting the crate in order to limit the electromagnetic interferences and still to allow the dissipation of the heat produced by the boards. The choice of a standard Ethernet protocol for communications makes the station independent of the type of outward link which then can be adapted to the communication networks available on the deployment site.

Emphasis was also put on improving the antenna design. A vertical butterfly shape was chosen to form a longer radiator (fig.10). The system is now presenting a very broad resonance around $60 \mathrm{MHz}$, an improved sensitivity with a spectrum clearly dominated by the galactic noise and a stronger rejection of AM and FM contributions. The Butterfly antenna shows nearly $3 \mathrm{~dB}$ between low and high galactic background levels when the galactic plane passes over Nançay. A fine calibration of the antenna response can thus be achieved by monitoring these variations. The new shape of the antenna allows an easier integration of the LNA at the heart of the radiators and an easier mechanical combination of two orthogonal antennas (i.e. dual polarization measurements). Thanks to the feature of the aluminum rod used an extremely fast shaping time is obtained for manufacturing the antenna, a very light and easy to assemble elements are produced.

Few prototype stations have been already produced and have been extensively tested (fig.10). Three of them will be installed by the end of the summer at Nançay and will be used in autonomous mode in concurrence with the existing wired antenna array. This minimalist network will allow the commissioning of the station in a self-triggering mode, the localization of the recorded transient signals and the analysis of the first events in coincidence with the antenna and particle detector arrays.

The production of the parts for assembling a hundred of autonomous stations is currently on the way. By the end of the year, a new set of 20 stations will be implemented within the current antenna array. On a short term scale, an additional extension phase of autonomous stations is considered at the Nançay observatory to increase further out the coverage of the CODALEMA experiment.

Finally few autonomous stations will be shipped and installed at the Pierre Auger Observatory within the frame of the AERA project [6].

\section{References}

[1] D.Ardouin et al. Nucl. Instrum. Meth. A555 (2005) 148

[2] D.Ardouin et al. Astro. Phys. 26 (2006) 341.

[3] D. Lebrun for the CODALEMA Collaboration, in: 30th International Cosmic Ray Conference on ICRC07, Merida, Mexico, 2007.

[4] D. Charrier, the CODALEMA Collaboration, in: IEEE Antennas and Propagation Society International Symposium, Honolulu, Hawai, June 1015, 2007, p. 4485.

[5] D.Ardouin et al., Astro. Phys. 31 (2009) 192

[6] R.Dallier et al., these proceedings. 